\title{
RNA sequencing reveals transcriptomic changes in tobacco (Nicotiana tabacum) following NtCPS2 knockdown
}

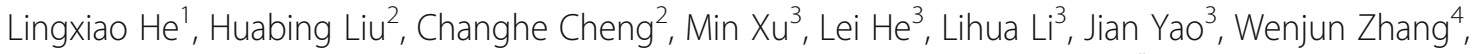
Zhengguang Zhai ${ }^{4}$, Qinzhan Luo ${ }^{5}$, Jutao Sun ${ }^{1}$, Tiezhao Yang ${ }^{1}$ and Shixiao Xu ${ }^{1 *}$

\begin{abstract}
Background: Amber-like compounds form in tobacco (Nicotiana tabacum) during leaf curing and impact aromatic quality. In particular, cis-abienol, a polycyclic labdane-related diterpenoid, is of research interest as a precursor of these compounds. Glandular trichome cells specifically express copalyl diphosphate synthase (NtCPS2) at high levels in tobacco, which, together with NtABS, are major regulators of cis-abienol biosynthesis in tobacco.

Results: To identify the genes involved in the biosynthesis of cis-abienol in tobacco, we constructed transgenic tobacco lines based on an NtCPS2 gene-knockdown model using CRISPR/Cas9 genome-editing technology to inhibit NtCPS2 function in vitro. In mutant plants, cis-abienol and labdene diol contents decreased, whereas the gibberellin and abscisic acid (ABA) contents increased compared with those in wild-type tobacco plants. RNA sequencing analysis revealed the presence of 9514 differentially expressed genes (DEGs; 4279 upregulated, 5235 downregulated) when the leaves of wild-type and NtCPS2-knockdown tobacco plants were screened. Among these DEGs, the genes encoding cis-abienol synthase, ent-kaurene oxidase, auxin/ABA-related proteins, and transcription factors were found to be involved in various biological and physiochemical processes, including diterpenoid biosynthesis, plant hormone signal transduction, and plant-pathogen interactions.
\end{abstract}

Conclusions: The present study provides insight into the unique transcriptome profile of NtCPS2 knockdown tobacco, allowing for a better understanding of the biosynthesis of cis-abienol in tobacco.

Keywords: CRISPR-Cas9, Nicotiana tabacum, RNASeq, Labdanoid diterpenes, cis-Abienol, Genome editing, Terpenoids

\section{Background}

Aroma is an important attribute of tobacco (Nicotiana tabacum L.) leaves. It is an indicator of tobacco quality and is influenced by a variety of chemical components [1]. An important aromatic substance in tobacco leaf surface secretions is cis-abienol, which belongs to the

\footnotetext{
* Correspondence: xushixiao@henau.edu.cn

${ }^{1}$ College of Tobacco Science, Henan Agricultural University, National Tobacco Cultivation \& Physiology \& Biochemistry Research Centre, Scientific

Observation and Experiment Station of Henan, Ministry of Agriculture, Zhengzhou 450002, China

Full list of author information is available at the end of the article
}

labdanoid diterpenoid family $[2,3]$. Previous studies have reported that cis-abienol plays an important role in determining the aromatic characteristics of tobacco, and it is an important precursor in the chemical synthesis of amber-like substances [4-6], which can affect aromatic quality. Furthermore, cis-abienol is involved in plant resistance to insects $[7,8]$ and diseases [9]. Therefore, it is important to explore the cis-abienol synthesis pathway in tobacco to better understand how to create diseaseresistant tobacco varieties with high-quality or characteristic aromas upon flue curing.

C C The Author(s). 2021 Open Access This article is licensed under a Creative Commons Attribution 4.0 International License, which permits use, sharing, adaptation, distribution and reproduction in any medium or format, as long as you give appropriate credit to the original author(s) and the source, provide a link to the Creative Commons licence, and indicate if changes were made. The images or other third party material in this article are included in the article's Creative Commons licence, unless indicated otherwise in a credit line to the material. If material is not included in the article's Creative Commons licence and your intended use is not permitted by statutory regulation or exceeds the permitted use, you will need to obtain permission directly from the copyright holder. To view a copy of this licence, visit http://creativecommons.org/licenses/by/4.0/ The Creative Commons Public Domain Dedication waiver (http://creativecommons.org/publicdomain/zero/1.0/) applies to the data made available in this article, unless otherwise stated in a credit line to the data. 
The biosynthesis of cis-abienol in tobacco was initially reported to be controlled by a single gene, $A b l[10,11]$, which is located on chromosome A [12]. Subsequently, Vontimitta et al. [13] used 117 doubled haploid lines and simple sequence repeat molecular markers to locate the genes regulating cis-abienol and sucrose ester accumulation and found that both genes are located on chromosome A. The genetic distance between two genes is $8.5 \mathrm{cM}$, and a total of 17 pairs of markers can be found in the linkage group. Among them, PT10324 and $A b l$ are completely separated. The markers beside $\mathrm{Abl}$ are PT55091 and PT61373, with distances of 2.02 and $0.6 \mathrm{cM}$, respectively [13]. Copalyl diphosphate synthase 2 (CPS2) from the angiosperm Cistus creticus subsp. creticus was first analysed through prokaryotic expression and dephosphorylation. Then, gas chromatography-mass spectrometry (GC-MS) analysis revealed that CPS2 catalyses the formation of 13(E)-labden-8-ol-15-diphosphate, implying that CPS2 is involved in the biosynthesis of cis-abienol [14]. In gymnosperms, cis-abienol synthase $(A B S / K S)$ contains both class I and class II functional domains, as shown by cloning and characterizing the gene from balsam fir (Abies balsamea) via transcriptome sequencing [15]. Sallaud et al. [16] cloned NtCPS2 and $N t A B S$ from tobacco and showed that both genes are involved in the biosynthesis of cis-abienol, which involves two steps. First, CPS-like catalytic activity yields 8hydroxy-copalyl diphosphate with a normal configuration, which can then be converted to cis-abienol by the $N t A B S$ product [16-18]. No other diterpenoid synthase has been reported to use 8-hydroxy-copalyl diphosphate as a substrate in dicotyledons to date. In addition, promoter analysis of NtCPS 2 showed that it could drive the expression of the GUS gene in glandular hairs [16, 19, 20]. The identification of NtCPS 2 and $N t A B S$ is of great significance for breeding high-quality tobacco and future microbial metabolic engineering. From this knowledge base, other diterpenoid-synthesising genes can be cloned and identified.

Among tobacco types, cis-abienol accumulates at different levels. It is mainly found in oriental and cigar tobacco but not in flue-cured tobacco, Burley tobacco, or Maryland tobacco $[1,16,21]$. To study the variation in cis-abienol content among different types of cultivated tobacco, 157 varieties of tobacco with or without cisabienol were selected, and the expression levels of $N t C P S 2$ and NtABS were analysed [16]. NtABS cDNA sequences did not differ among tobacco varieties, but two distinct polymorphisms were found in NtCPS2 cDNA: an 8-bp insertion at position 275 and a G-T transversion at position 292 of NtCPS2. Both of these result in a stop codon, which leads to early termination and shortening of the encoded peptide chain. Because the encoded protein loses its active site, it also loses its original function [16]. Thus, NtCPS2 is key for cis-abienol biosynthesis. However, the mechanism by which the metabolic pathway of labdanoid diterpenoids is influenced by NtCPS 2 in tobacco and the effects of NtCPS2 knockdown on other metabolic pathways are still unknown.

In this study, we used CRISPR/Cas9 gene-editing technology to knock down NtCPS2. The CRISPR/Cas9 $N t C P S 2$ expression vector was constructed from the high-aroma strain 8306 and transformed NtCPS2-knockdown plants were obtained. A high-throughput RNA sequencing (RNA-seq) technique was used to compare expression profiles between mutant and 8306 plants. Sequencing results were verified using fluorescence quantitative polymerase chain reaction (PCR), and physiological changes and transcriptional inheritance were analysed. By elucidating the function of the NtCPS2 gene and the molecular mechanisms underlying the influence of its related genes, high-aroma tobacco varieties can be cultivated.

\section{Results \\ Targeted mutagenesis of NtCPS2 by CRISPR/Cas9 in tobacco}

To generate Cas9-induced mutations in NtCPS2, a vector was designed that harboured chimaeric guide RNA (gRNA) to guide Cas9 to target sequences where it bound and cleaved genomic DNA to generate doublestrand breaks [22]. Two target sites of CPS2 were selected (Supplementary Figure 1). The gRNA for each target site was generated by overlap-extension PCR. Cas9 was subcloned into a single expression vector [23]. The Cas9 and gRNA expression cassette was located in one expression vector (pRGEB32-Cas9-NPT II-CPS2-gRNA). Through the Agrobacterium tumefaciens-mediated method, 36 transformed regenerated plants in the $\mathrm{T}_{0}$ generation were obtained. After amplification with target-specific primers, all positive samples were sequenced to assess the mutation efficiency. Of 36 plants, eight were transgenic lines. Most of the transgenic lines had a single-base insertion of $\mathrm{A}, \mathrm{C}$, or $\mathrm{T}$ at Target 2 . Thus, as the peptide chain was formed, the stop codon was encountered early in the process, and the translated amino acid chain was greatly shortened. To test the heritability of the mutations, homozygous transgenic plants in the $T_{0}, T_{1}$, and $T_{2}$ generations were analysed. Detailed information about the homozygous $\mathrm{T}_{2}$ plants (M1-M9) is shown in Fig. 1A, and these plants were used for the following experiments.

\section{NtCPS2 knockdown affects cis-abienol content}

To verify whether the gene mutations caused changes in gene expression, quantitative real-time PCR (qRT-PCR) was used to detect the expression levels of NtCPS 2 in 


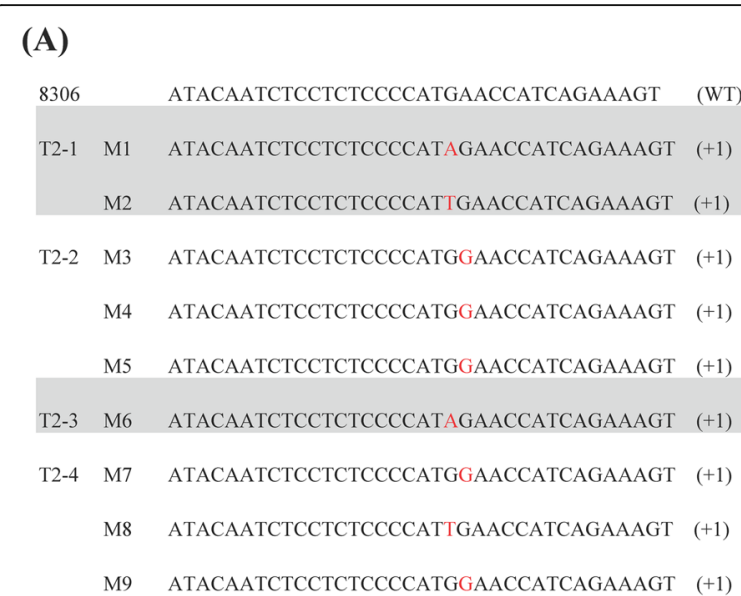

(B)

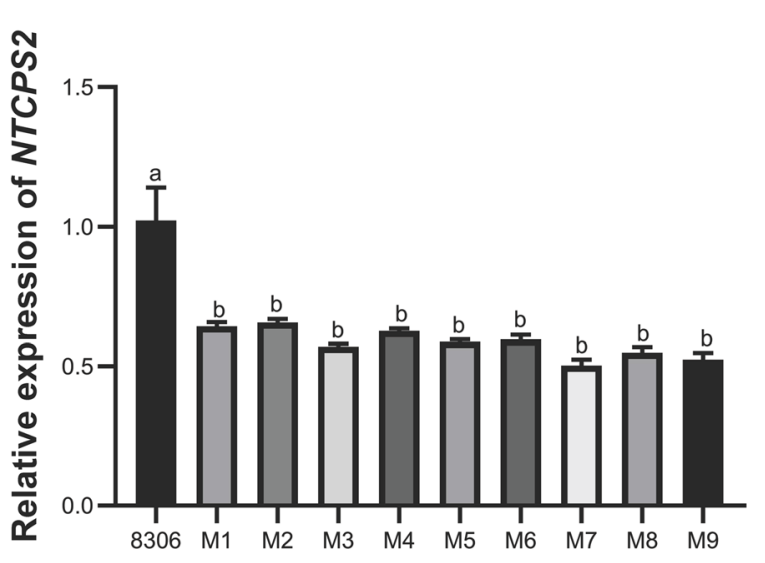

(C)

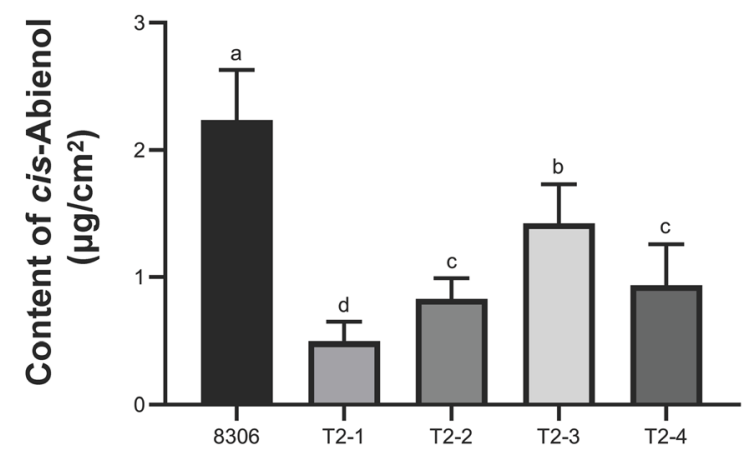

Fig. 1 CRISPR/Cas9-induced mutations in T2 transgenic tobacco plants. A Sequences reflecting targeted inversions in the mutants. B Relative expression of NTCPS2 in the mutants. C Quantification of cisabienol in homozygous $\mathrm{T} 2$ transgenic tobacco plants. Values are averages of at least three different plants or three different leaves

the leaves of mutant and wild-type (8306) plants. The results showed that NtCPS2 expression decreased significantly in transgenic plants compared to wild-type plants (Fig. 1B). To detect changes in cis-abienol content in the leaves, exudates were collected from the mutant plants and analysed using GC-MS. The contents of cis-abienol also decreased significantly in mutant plants compared to wild-type plants (Fig. 1C). The results indicate that $N t C P S 2$ is one of the key genes regulating the cis-abienol biosynthesis pathway, and NtCPS2 knockdown results in low levels of cis-abienol biosynthesis and accumulation. $N t A B S$ is another key gene involved in cis-abienol biosynthesis [16]. A previous study reported that cis-abienol was detected in plants expressing both NtCPS2 and $N t A B S$ but not in plants expressing just one of the two genes [16]. NtABS expression was weak in the mutant plants compared to the wild-type plants, implying that $N t C P S 2$ knockdown negatively influenced NtABS expression. This is possibly because NtCPS2 is located upstream of $N t A B S$ in the cis-abienol biosynthesis pathway.

\section{NtCPS2 has a minor effect on the development of glandular trichomes in tobacco}

Agronomic characteristics were analysed to assess the mutant phenotypes (Fig. 2 and Supplementary Figure 2). Differences in plant height, internode length, number of leaves, and stem girth between mutant and wild-type plants did not exhibit the same trend. T2-2 mutants had longer internodes and wider stems than other mutants and wild-type plants, whereas all mutants except for T2-1 had shorter plant heights than the wild-type plants (Supplementary Figure 2). These results indicate that NtCPS2 expression does not strongly affect tobacco plant morphology. As NtCPS2 is specifically expressed in glandular cells [16], the morphology of the glandular trichomes on the largest leaf of each plant was examined. Both the length and width of the largest leaf were significantly shorter in mutant plants than in wild-type plants. The average diameter of glandular trichomes was smaller in mutant plants, especially $\mathrm{T} 2-1$, whereas both longer and shorter glandular trichomes were observed in mutant plants compared to wild-type plants (Fig. 2). Other trichome characteristics, such as the numbers of long and short trichomes, did not differ significantly between mutant and wild-type plants (data not shown). Thus, in the absence of NtCPS2 expression in tobacco plants, the diameter of glandular cells and the area of the largest leaf decrease, but not the length of glandular trichomes. The T2-1 line was selected and used to profile transcriptomic changes after NtCPS2 knockdown in tobacco 8306.

\section{Overview of transcriptome sequencing}

To profile gene expression after NtCPS2 knockdown, RNA-seq libraries were constructed for the mutant and wild-type plants. Six samples of each line were sequenced, and $41.64 \mathrm{G}$ of clean data was obtained. In total, 6.70-7.02 G of effective data was collected from each sample, with a Q30 distribution of 94.29-94.91\% and an average GC content of $43.41 \%$. More than 
(A)

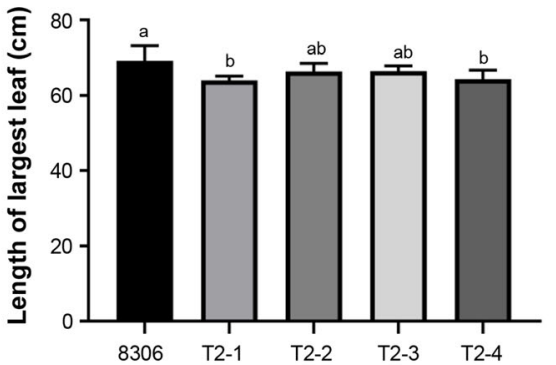

(C)

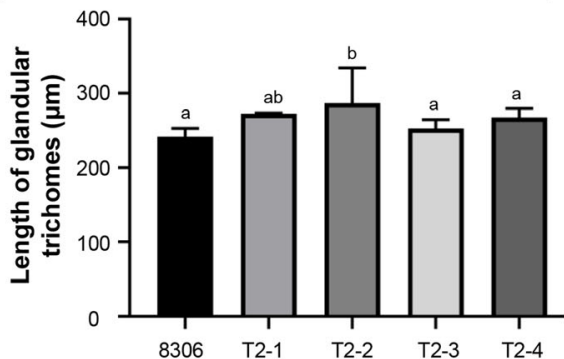

(B)

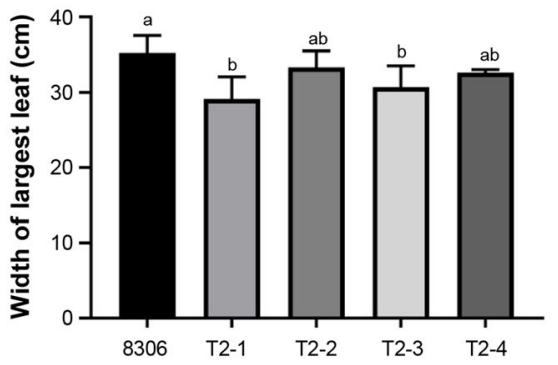

(D)

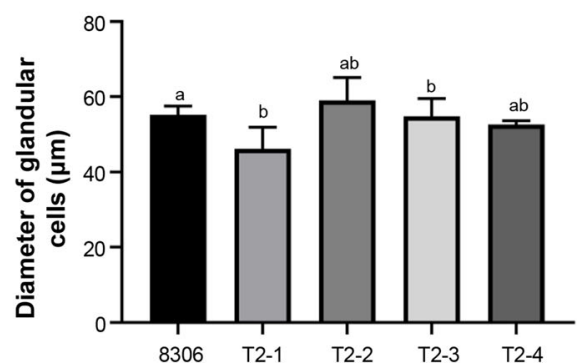

Fig. 2 Morphological characteristics of mutant and wild-type plants, including the length (A) and width (B) of the largest leaf, length of glandular trichomes (C), and diameter of glandular cells (D). Values are presented as the means \pm standard deviations $(n=4$ for leaves and $n=100$ for glandular trichomes). Different lowercase letters denote significant differences among plant lines $(p<0.05)$

95.58\% of the clean reads had quality scores that met the Q30 criterion (probability of base-calling error = $0.1 \%$ ) [24]. Furthermore, the GC content ranged from 43.15 to $43.66 \%$. The sequencing data are summarized in Table 1 .

\section{Analysis of differentially expressed genes (DEGs) and their functions}

Volcano plots were used to assess the variation in gene expression between mutant and wild-type plants (Fig. 3A). In total, 9514 DEGs were detected. Among them, 4279 were upregulated and 5235 were downregulated in the transgenic tobacco plants compared to 8103 using the thresholds $p<0.05$ and $\mid \log _{2}$ (fold change $[\mathrm{FC}]) \mid>1$ (Fig. 3B).

Kyoto Encyclopedia of Genes and Genomes (KEGG) and Gene Ontology (GO) pathway analyses of the differentially expressed mRNAs were performed to determine the functions of the DEGs. The 20 most significantly enriched pathways (lowest $q$ values) according to KEGG metabolic pathway annotation were examined in detail (Fig. 4A). Based on GO analysis, the DEGs were most likely to be associated with biological processes (Fig. 4B) and cellular components (Fig. 4C). A large percentage of the DEGs were assigned to the categories metabolic process, cellular process, catalytic activity, binding, and single-organism process, with only a few genes assigned to channel regulator activity, cell killing, and protein tag. The DEGs involved in the pathways for diterpenoid biosynthesis, plant hormone signal transduction, and plantpathogen interactions were analysed in detail.

\section{Validation of selected DEGs using qRT-PCR}

To validate the RNA-seq data, 12 DEGs, including genes involved in cis-abienol and gibberellin (GA) biosynthesis as well as genes related to plant-pathogen interactions

Table 1 Summary of RNA-sequencing outcomes

\begin{tabular}{lllllll}
\hline Sample & Raw reads & Clean reads & Raw bases & Clean bases & Q30 (\%) & GC (\%) \\
\hline Con1 & $49.05 \mathrm{M}$ & $47.90 \mathrm{M}$ & $7.36 \mathrm{G}$ & $6.89 \mathrm{G}$ & 94.60 & 43.46 \\
Con2 & $49.76 \mathrm{M}$ & $48.74 \mathrm{M}$ & $7.46 \mathrm{G}$ & $7.02 \mathrm{G}$ & 94.88 & 43.25 \\
Con3 & $47.66 \mathrm{M}$ & $46.51 \mathrm{M}$ & $7.15 \mathrm{G}$ & $6.70 \mathrm{G}$ & 94.29 & 43.30 \\
L1 & $49.71 \mathrm{M}$ & $48.57 \mathrm{M}$ & $7.46 \mathrm{G}$ & $6.97 \mathrm{G}$ & 94.67 & 43.66 \\
L2 & $49.77 \mathrm{M}$ & $48.72 \mathrm{M}$ & $7.47 \mathrm{G}$ & $6.99 \mathrm{G}$ & 94.88 & 43.41 \\
L3 & $49.70 \mathrm{M}$ & $48.62 \mathrm{M}$ & $7.45 \mathrm{G}$ & $6.97 \mathrm{G}$ & 94.85 & 43.47 \\
\hline
\end{tabular}

Not: Con, wild type; L, mutant 
(A)

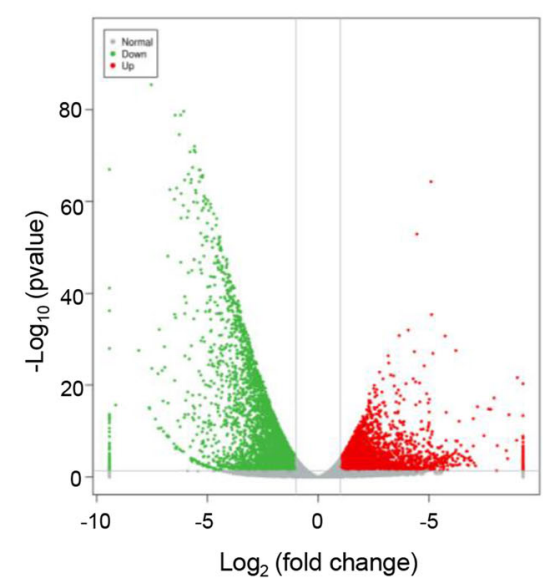

(B)

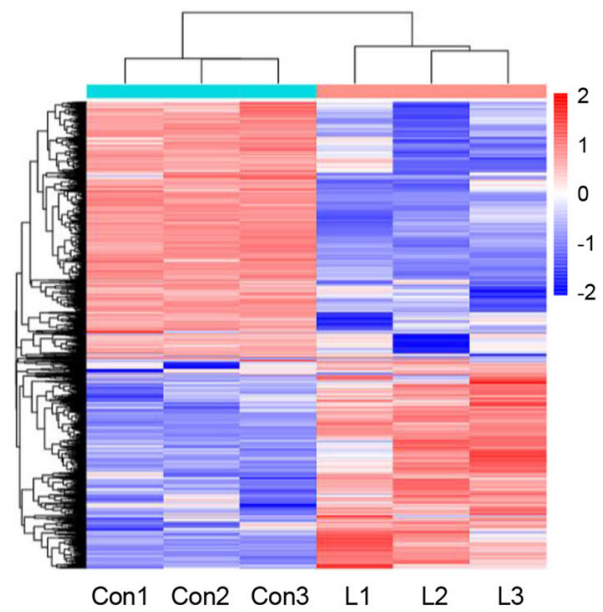

Fig. 3 Differentially expressed genes (DEGs) were screened using an absolute $\log _{2}(\mathrm{FC})$ value $>1$ and $p$-value $<0.05$. Significant differences in expression were observed for 9514 genes, as represented by a volcano plot (A) and heat map (B)

and other hormone signalling pathways, were selected randomly for $\mathrm{qRT}-\mathrm{PCR}$ analysis. The gene expression patterns determined using qRT-PCR were consistent with those determined via transcriptome sequencing (Fig. 5). FC values differed between qRT-PCR and RNAseq, possibly due to differences in the sensitivity of each method or because different samples were used for qRTPCR and RNA-seq.

\section{Expression levels of genes related to cis-abienol biosynthesis decreased significantly in mutant plants} NtCPS2 (Nitab4.5_0001630g0010) was identified as a DEG via RNA-seq, and its expression level was 9.27-fold lower in the mutant than in the wild type. The expression level of another key gene related to cis-abienol biosynthesis, NtABS (Nitab4.5_0015240g0010), also decreased 2.43-fold in the mutant. NtCPS2 and NtABS operate in succession to synthesize cis-abienol [16]. When both NtCPS2 and NtABS are expressed, cis-abienol is synthesized and can be detected in plants. However, cisabienol synthesis does not occur in plants that express only one of these genes [16]. NtCPS2 encodes 8hydroxy-copalyl diphosphate synthase, which synthesizes 8-hydroxy-copalyl diphosphate, and NtABS encodes a kaurene synthase-like (KSL) protein, abienol synthase, which uses 8-hydroxy-copalyl diphosphate to produce cis-abienol. Our results indicate that weak expression of $N t C P S 2$ directly or indirectly results in a decrease in the expression level of $N t A B S$ and, consequently, low cisabienol contents. Another putative cis-abienol synthase (Nitab4.5_0008024g0010) was also found to be downregulated in the mutant, indicating that this enzyme may have the same substrate as $N t A B S$ and thus be involved in the cis-abienol biosynthesis pathway. In contrast, other putative cis-abienol synthases, including Nitab4.5_ 0004164g0070 and Nitab4.5_0004164g0010, were found to be upregulated in the mutant. These two enzymes may have other functions in tobacco. Other DEGs involved in the cis-abienol biosynthesis pathway were also identified (based on KEGG analysis) and had lower expression levels in the mutant (Fig. 4). This included KSL4 (Nitab4.5_0000029g0200, FC = 2.43) and genes predicted to encode ent-kaur-16-ene synthase (Nitab4.5_ 0002280g0060, FC = 5.61; and Nitab4.5_0002862g0030, $\mathrm{FC}=1.57$ ). As with KSL4, NtABS is a KSL gene (Table 2). Hence, KSL4 and genes that putatively encode ent-kaur16-ene synthase may be involved in cis-abienol biosynthesis. This needs to be verified in future work.

\section{GA biosynthesis increased significantly in mutant plants} According to diterpenoid biosynthesis pathways, the same substrate, geranylgeranyl pyrophosphate (GGPP), is used for cis-abienol and GA synthesis. In this study, most of the DEGs involved in GA biosynthesis were strongly upregulated in the mutant, including KAO2 (Nitab4.5_0001476g0100, FC=16.67), KS1 (Nitab4.5_ 0004164g0010, $\mathrm{FC}=2.76$ ), and CPS1 (Nitab4.5_ 0010312g0010, FC = 12.14) (Table 2). From these genes, ent-copalyl diphosphate synthase 1 (encoded by CPS1) and ent-kaurene synthase (encoded by KS1) were found to separately catalyse the synthesis of ent-kaurene from GGPP. However, KO (encodes ent-kaurene oxidase, which converts ent-kaurene to kaur-16-en-18-oate) expression was downregulated in the mutant. DEGs participating in the latter stages of the pathway, such as $K A O 2$ and $G A 20_{O X 2}$, were upregulated compared to the wild-type plants. $\mathrm{KO}$ and KAO belong to the CYP701A, P450, and CYP88A clades. Accordingly, KAO is localized 
(A)

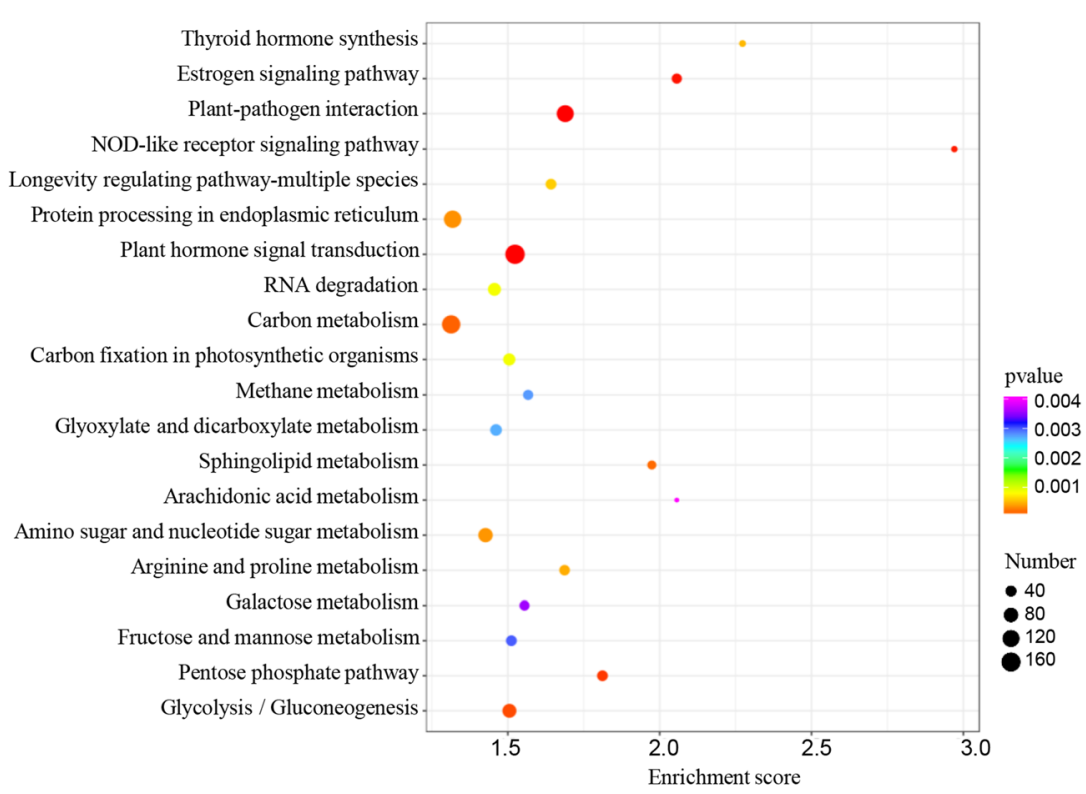

(B)

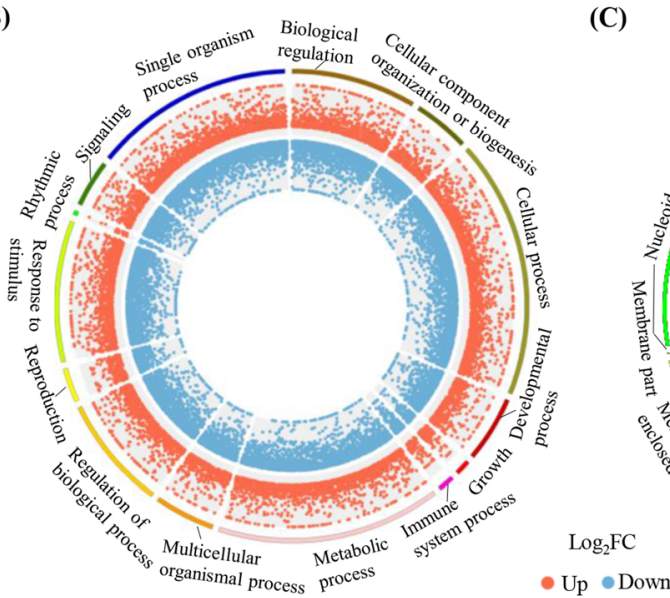

(C)

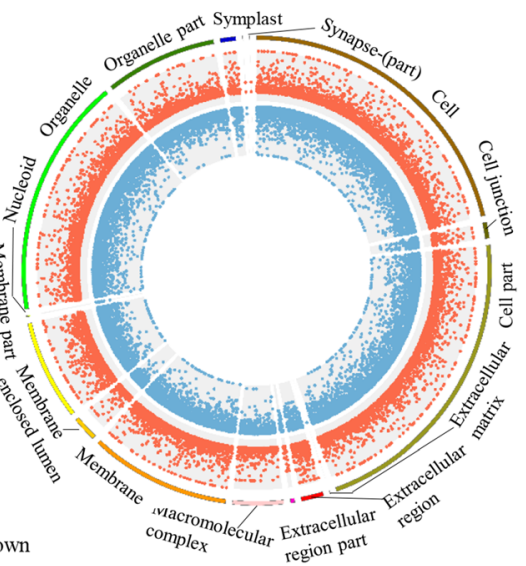

Fig. 4 Kyoto Encyclopedia of Genes and Genomes (KEGG) and Gene Ontology (GO) pathway analyses of DEGs after NtCPS2 knockdown. A The top 20 enrichment scores for KEGG pathway enrichment analysis of differentially expressed mRNAs. B GO annotations of DEGs assigned to the biological processes category. C GO annotations of DEGs assigned to the cellular components category

in the endoplasmic reticulum, whereas $\mathrm{KO}$ is localized in both the endoplasmic reticulum and plastid envelope [25]. The differential expression of $\mathrm{KO1}$ and $\mathrm{KAO2}$ in response to NtCPS2 knockdown was explored further. GA contents in mutant plants were also analysed via GCMS. The results showed that the GA contents in transgenic plants were significantly higher than those in wildtype plants (Fig. 6). GA12 is considered the precursor of all GAs in plants [26], and other GA forms are produced through oxidative steps catalysed by GA12. Genes involved in the production of these GA forms were upand downregulated in the mutants.

\section{Changes in abscisic acid (ABA) biosynthesis and signal transduction in mutant plants}

In carotenoid biosynthesis pathways, GGPP is also a substrate for ABA synthesis. RNA-seq analysis showed that four PSY genes (encoding phytoene synthases) were upregulated at the first step, which involves GGPP, in the mutant compared to the wild type. PSY is a transferase enzyme that is involved in the biosynthesis of carotenoids. It catalyses the conversion of GGPP to phytoene. Two genes encoding LCYs (lycopene epsilon cyclases) were also upregulated in the mutant at the next step. These results indicate that NtCPS 2 knockdown positively affects ABA synthesis, likely because substrate competition decreases. In addition, two ABA 8'-hydroxylases, which are involved in ABA degradation, were 


\section{(A) cis-Abienol biosynthesis}

CPS2

KSL 4 (Nitab4.5_0000029g0200)
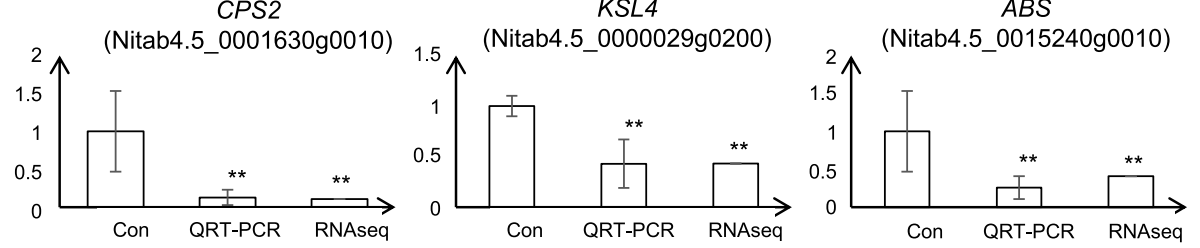

(B) Gibberellin biosynthesis
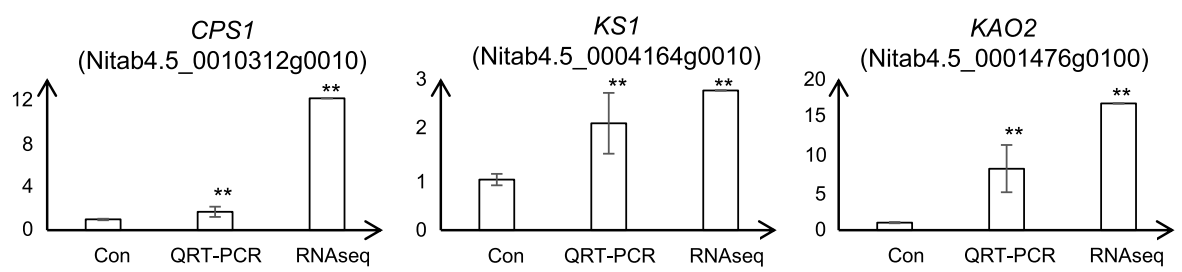

(C) Plant-pathogen interaction
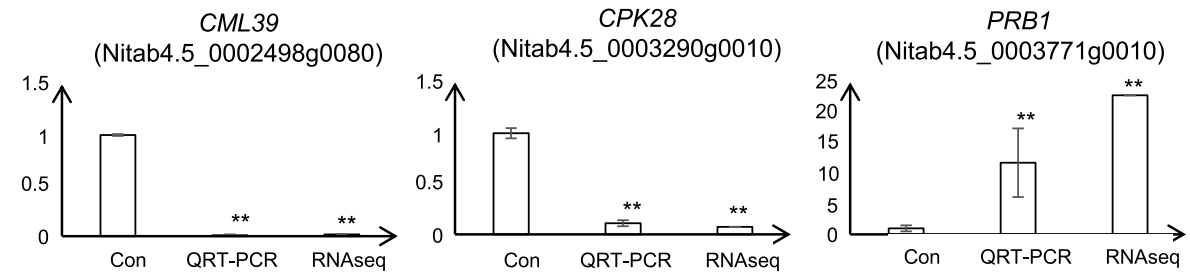

(D) Other hormone signalling-related genes
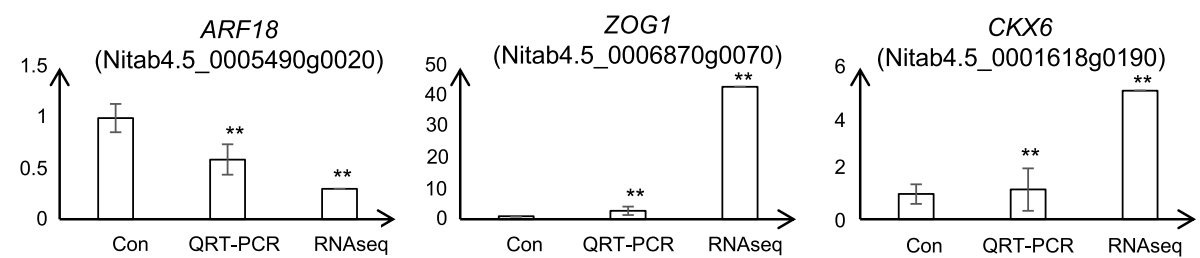

Fig. 5 Transcription profiles of selected genes in mutant and wild-type plants as determined using RNA-seq and quantitative real-time polymerase chain reaction (qRT-PCR). Relative transcription levels of DEGs involved in cis-abienol biosynthesis (A), gibberellin (GA) biosynthesis (B), plant-pathogen interactions (C), and other hormone-signalling pathways (D). Asterisks indicate significant differences between samples and the control $(p<0.05$, two-sample $t$-test $)$

downregulated in the mutant. In the ABA signal transduction pathway, five of six ABA receptors (PYLs), which inhibit the expression of protein phosphatase $2 \mathrm{C}$, were upregulated in the mutant. In the next step, serine/ threonine-protein kinase expression was upregulated in the mutant. This might have been related to stress responses and stomatal opening and closure in tobacco leaves.

Transcriptomic analysis of genes involved in plantpathogen interactions

In plants, cis-abienol may participate in insect resistance and disease resistance [27]. Plant resistance to pathogen attack can induce the accumulation of pathogenesisrelated proteins (PRs) that contribute to systematically acquired resistance. In this study, PRs were identified through RNA-seq. Of the 17 PRs, 14 (82.35\%) were significantly upregulated in the mutant compared with the wild type, including genes that encode PR proteins $1 \mathrm{~A}$, $\mathrm{B}$, and $\mathrm{C}$ (Table 3). Among the 17 families of PRs, PR 15, 9-11 and 17 were related to the acquisition of defence against pathogen infections. In addition, calcium is involved in regulating diverse physiological processes as a second messenger [28]. The results of transcriptomic analysis revealed that 15 of 19 CDPKs and most CAM/ CML were significantly downregulated upon NtCPS2 knockdown and a low content of cis-abienol, which disturbed the balance among active oxygen species, including rubidium hydroxide, reactive oxygen species, and nitric oxide synthase. Furthermore, the resistance of transgenic tobacco plants to black shank, induced by Phytophthora nicotianae, was checked. After the 
Table 2 Genes related to diterpenoid biosynthesis that are differentially expressed between NtCPS2-knockout and 8306 plants

\begin{tabular}{llll}
\hline Gene name & Gene ID & log $_{2}$ FC & Protein properties \\
\hline CPS2 & Nitab4.5_0001630g0010 & -3.21 & PREDICTED: copal-8-ol diphosphate hydratase, chloroplastic \\
KSL4 & Nitab4.5_0000029g0200 & -1.21 & PREDICTED: ent-kaur-16-ene synthase, chloroplastic isoform X3 \\
DLO2 & Nitab4.5_0000129g0310 & -2.53 & PREDICTED: gibberellin 2-beta-dioxygenase 8-like \\
GA2OX2 & Nitab4.5_0000222g0140 & -5.09 & PREDICTED: gibberellin 2-beta-dioxygenase 2 \\
GA2OX1 & Nitab4.5_0000923g0050 & -3.82 & PREDICTED: gibberellin 2-beta-dioxygenase 1-like \\
GA2OX2 & Nitab4.5_0001013g0080 & -2.88 & PREDICTED: gibberellin 2-beta-dioxygenase 2-like \\
KAO2 & Nitab4.5_0001476g0100 & 4.06 & PREDICTED: ent-kaurenoic acid oxidase 1-like isoform X2 \\
GA200X2 & Nitab4.5_0001573g0060 & 2.08 & gibberellin 20 oxidase 1-like \\
GA2OX1 & Nitab4.5_0002209g0240 & -1.46 & gibberellin 2-beta-dioxygenase 1-like \\
KO & Nitab4.5_0002280g0060 & -2.49 & PREDICTED: ent-kaurene oxidase, chloroplastic \\
GA2 & Nitab4.5_0002862g0030 & -1.19 & PREDICTED: ent-kaur-16-ene synthase, chloroplastic-like isoform X1 \\
KS1 & Nitab4.5_0004164g0010 & 1.46 & PREDICTED: cis-abienol synthase, chloroplastic-like \\
TPS1 & Nitab4.5_0004164g0070 & 3.00 & PREDICTED: cis-abienol synthase, chloroplastic-like \\
GA2 & Nitab4.5_0008024g0010 & -1.20 & PREDICTED: cis-abienol synthase, chloroplastic-like \\
CPS1 & Nitab4.5_0010312g0010 & 3.60 & PREDICTED: ent-copalyl diphosphate synthase, chloroplastic-like isoform X1 \\
ABS & Nitab4.5_0015240g0010 & -1.28 & cis-abienol synthase, chloroplastic \\
\hline FC fold & &
\end{tabular}

FC fold change

treatment of $P$. nicotianae for 7 days, wild-type plants showed wilting symptoms, while the transgenic tobacco plants were not (Supplementary Figure 3).

\section{Discussion}

The aromatic characteristics of tobacco are improved by cis-abienol, which belongs to the labdane diterpenoid family. Although the genes encoding the enzymes participating in the two steps of cis-abienol biosynthesis have been cloned in tobacco [16], the function and transcriptome profile of NtCPS2 knockdown are less well understood. By knocking down NtCPS2, whose product catalyses the first reaction in the cis-abienol biosynthesis pathway, we were able to examine how cis-abienol

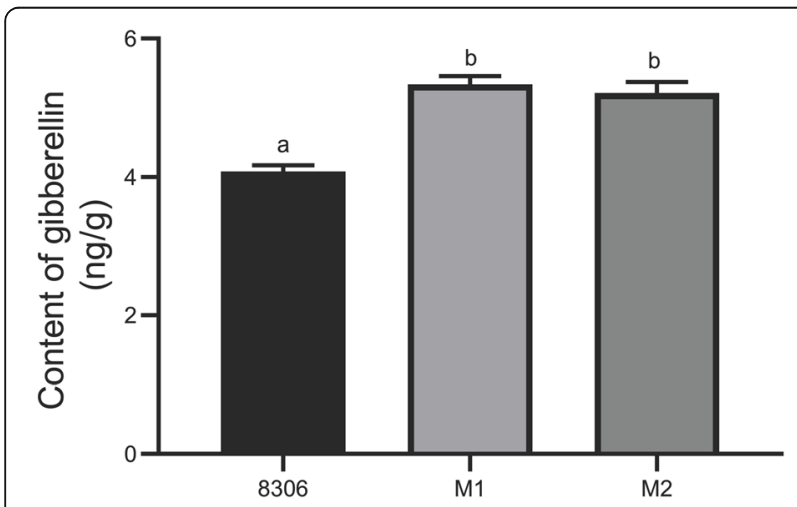

Fig. 6 Gibberellin contents in the leaves of NtCPS2-knockdown (M1 and M2) and wild-type (8306) tobacco. Different lowercase letters denote significant differences among plant strains $(p<0.05)$ biosynthesis and other related metabolic pathways are controlled. The regulatory network is shown in Fig. 7.

NtCPS2 plays a limited role in the biosynthesis of cisabienol and other terpenoids

Mutations in NtCPS 2 were previously reported to be strongly correlated with the absence of cis-abienol and labdene-diol in tobacco or a decrease in their levels [16]. In $N$. sylvestris, cis-abienol accumulates when both $N t C P S 2$ and $N t A B S$ are expressed [16]. In this study, we generated NtCPS2-knockdown tobacco lines using the CRISPR-Cas9 method. In mutant plants that weakly expressed NtCPS2, the levels of cis-abienol produced decreased (Fig. 1). NtABS is involved in the second step of cis-abienol biosynthesis, and its expression levels also decreased. The decreased expression of both of these genes might have resulted in low levels of the intermediate 8hydroxy-copalyl diphosphate accumulating and the cessation of cis-abienol production downstream (Fig. 1C). The results indicate that NtCPS2 plays a key role in cisabienol biosynthesis; thus, downregulated gene expression leads to inactivation of the cis-abienol biosynthesis pathway.

The precursor GGPP, which participates in the first step of the pathway, is a common precursor for the biosynthesis of not only diterpenoids (including cis-abienol and labdene-diol) but also GA, carotenoids (including ABA), and the phytolchain of chlorophyll [29]. When NtCPS2 is absent, GGPP is not catalysed to produce 8hydroxy-copalyl diphosphate, and other reactions that use GGPP as a substrate are enhanced. During GA 
Table 3 Properties of DEGs encoding pathogenesis-related proteins

\begin{tabular}{llll}
\hline Gene name & Gene ID & log $_{\mathbf{2}}$ FC & Protein properties \\
\hline PRB1 & Nitab4.5_0003771g0010 & 4.52 & Pathogenesis-related protein 1A \\
OSM34 & Nitab4.5_0004097g0050 & 3.76 & PREDICTED: pathogenesis-related protein R minor form \\
PRB1 & Nitab4.5_0014031g0010 & 3.51 & PREDICTED: pathogenesis-related protein 1B-like \\
- & Nitab4.5_0006088g0020 & 3.46 & PREDICTED: pathogenesis-related protein PR-4B \\
- & Nitab4.5_0018960g0010 & 3.35 & PREDICTED: pathogenesis-related protein PR-4B \\
- & Nitab4.5_0008835g0020 & 3.27 & PREDICTED: pathogenesis-related protein STH-2-like \\
- & Nitab4.5_0004861g0030 & 3.24 & PREDICTED: pathogenesis-related protein 1C-like \\
PRB1 & Nitab4.5_0004861g0040 & 3.16 & PREDICTED: pathogenesis-related protein 1C \\
- & Nitab4.5_0008375g0050 & 3.06 & PREDICTED: pathogenesis-related protein STH-2-like \\
HEL & Nitab4.5_0009495g0020 & 2.73 & PREDICTED: pathogenesis-related protein PR-4A \\
TL1 & Nitab4.5_0008011g0010 & 2.29 & PREDICTED: pathogenesis-related protein 5-like isoform X1 \\
- & Nitab4.5_0000194g0120 & 2.17 & PREDICTED: pathogenesis-related protein STH-2-like \\
CRF2 & Nitab4.5_0000105g0290 & 2.06 & PREDICTED: pathogenesis-related genes transcriptional activator PTI6-like \\
CRF2 & Nitab4.5_0002902g0060 & 1.11 & PREDICTED: pathogenesis-related genes transcriptional activator PTI6-like \\
MOS11 & Nitab4.5_0002073g0060 & -1.47 & PREDICTED: pathogenesis-related protein PRMS-like \\
CRF2 & Nitab4.5_0000586g0010 & -2.67 & PREDICTED: pathogenesis-related genes transcriptional activator PTI6-like \\
CRF2 & Nitab4.5_0007730g0010 & -2.72 & PREDICTED: pathogenesis-related genes transcriptional activator PTI6-like \\
\hline & &
\end{tabular}

biosynthesis in Arabidopsis, GGDP is converted to entkaurene in a two-step reaction catalysed by CPS and KS, which are encoded by $A t C P S$ and AtKS, respectively [30, 31]. In this study, NtCPS1 and NtKS1 expression levels were upregulated after NtCPS2 knockdown, and GA production, which occurs downstream, increased in the leaves of mutant plants (Fig. 6). In terms of carotenoid biosynthesis, genes (including phytoene synthase 2 and lycopene epsilon cyclase) involved in converting GGPP to phytoene were upregulated after NtCPS2 knockdown. Overall, reactions that consume GGPP as a substrate were enhanced. The results indicate that NtCPS2

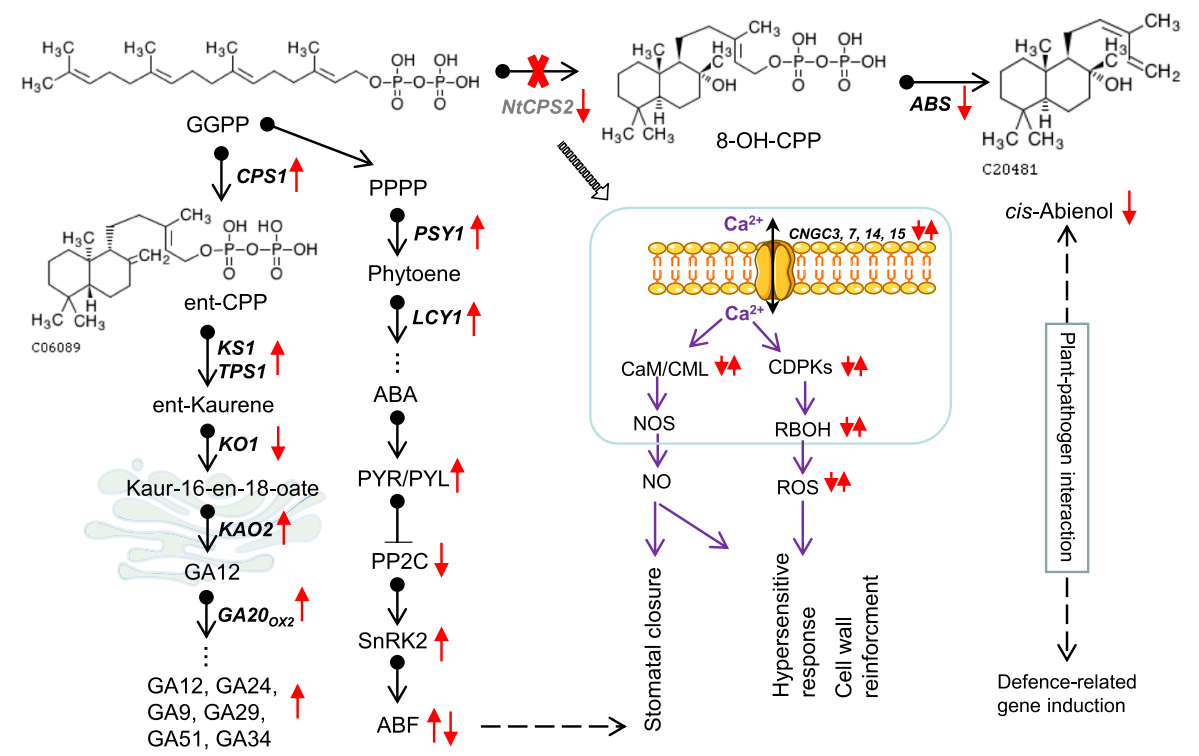

Fig. 7 The changes in metabolic pathways associated with NtCPS2 knockdown in tobacco mutants derived from KEGG database. 8-OH-CPP, 8hydroxy-copalyl diphosphate; ABA, abscisic acid; ABS, cis-abienol synthase; CDPK, calcium-dependent protein kinase; CPS1, copalyl diphosphate synthase 1; GGPP, geranyl diphosphate; LCY1, lycopene epsilon cyclase 1; NO, nitric oxide; NOS, nitric oxide synthase; PP2C, protein phosphatase 2C; PPPP, prephytoene diphosphate; PSY1, phytoene synthase 1; RBOH, rubidium hydroxide; ROS, reactive oxygen species; SnRK2, serine/ threonine-protein kinase 2 
knockdown also contributes to the biosynthesis of other terpenoids depending on the same substrate. Future studies can verify this hypothesis by overexpressing $N t C P S 2$ and/or NtABS.

\section{Plants with mutations in NtCPS2 still exhibit wild-type morphology}

Diterpenoids such as cembranoid diterpenes and labdanoid diterpenes from tobacco-leaf exudates significantly influence cigarette smoke characteristics and flavour profiles [2, 3]. To our knowledge, no previous studies on the effects of cis-abienol on the growth and development of tobacco plants have been reported. We found that the mutant and wild-type morphology did not differ much, except for the diameter of the glandular trichomes. This indicates that NtCPS 2 knockdown and the subsequent decrease in cis-abienol do not affect tobacco plant morphology. However, the contents of other chemical substances (including GA and ABA) may change in mutants. Mutants had higher levels of GA and did not exhibit GA-overdose morphology. In Arabidopsis, CPSand/or KS-overexpressing mutants also did not exhibit GA-overdose morphology [32]. This suggests that the levels of bioactive GA in these plants likely did not change. Transcriptomic analysis showed that the expression of $\mathrm{GA} 20_{\mathrm{OX} 2}$ was upregulated, whereas that of $\mathrm{GA} 2_{\mathrm{OX} 4}, \mathrm{GA} 2_{\mathrm{OX} 2}$, and $\mathrm{GA} 2_{\mathrm{OX} 1}$ was downregulated (Table 2). Wild-type Arabidopsis plants treated with exogenous GA and transgenic plants overexpressing the downstream GA biosynthesis gene AtGA20 oxl both exhibited aspects of GA overdose morphology [33]. The differential regulation of $\mathrm{GA} 20_{\mathrm{OX}}, \mathrm{GA} 2_{\mathrm{Ox} 4}, \mathrm{GA} 2_{\mathrm{OX} 2}$, and GA2 $2_{\mathrm{Ox} 1}$ might result in different types of GA accumulating at different levels; thus, overall levels of bioactive GA may not change much, and plants may not exhibit a GA-underdose morphology.

\section{cis-abienol may participate in tobacco disease resistance} Labdanoid diterpenes may exhibit defence-related activities such as antifungal [9] and insecticidal [7, 8] activities [27, 34]. The application of cis-abienol to the roots of tobacco, tomato, and Arabidopsis at a concentration of $100 \mu \mathrm{mol} / \mathrm{L}$ can induce the expression of resistance genes and inhibit bacterial wilt disease [27]. In vitro experiments showed that concentrations of cis-abienol and related diterpenoids in the range of $0.01-100 \mathrm{ppm}$ can inhibit the growth of Phytophthora nicotianae in tobacco [35]. However, cis-abienol isolated from Cunninghamia konishii had no inhibitory effect on the growth of wood decay fungi [36]. Kennedy et al. [37] found that the concentration of cis-abienol from $3.75 \times 10^{-4} \mu \mathrm{g} / \mathrm{cm}^{2}$ to $120 \mu \mathrm{g} / \mathrm{cm}^{2}$ had inhibitory effect on Peronospora tabacina. Compared with the control, the germination of sporangia was not affected by 10 kinds of cis-abienol concentrations. At the two lowest concentrations, $3.75 \times$ $10^{-4} \mu \mathrm{g} / \mathrm{cm}^{2}$ and $3.75 \times 10^{-3} \mu \mathrm{g} / \mathrm{cm}^{2}$, the incidence rate of Peronospora tabacina in tobacco was higher than that in the control group. In this study, the resistance of NtCPS 2 mutant tobacco lines to black shank was better than that of wild-type plants. Under field conditions, cisabienol does not have an effect on diseased leaves in tobacco. QTL Phn15.1 in the cigar tobacco cultivar Beinhart 1000 was discovered, which provides a high level of partial resistance to black shank disease caused by $P$. nicotianae [13, 38]. A very close genetic association was found between Phn15.1 and the ability to biosynthesize cis-abienol. Recently, Steede et al. [35] observed no correlation between field resistance to $P$. nicotianae and the ability to accumulate cis-abienol in either transgenic materials or mapping populations. Cis-Abienol has little effect on black shank disease development under natural field conditions. Therefore, whether the accumulation of cis-abienol and the genes related to cis-abienol synthesis contributed to resistance against $P$. nicotianae in tobacco needs to be explored further. In this study, NTCP $S 2$ was edited to construct transgenic materials with low cis-abienol content. It was found that the transgenic materials were sick later than the wild-type in response to Phytophthora nicotianae treatment. In the subsequent experiments, we found that the ratio of $\mathrm{GA}_{3} / \mathrm{ABA}$ changed, which may have an impact on the resistance of transgenic materials.

A key defence response to pathogen attack in plants is the induction and accumulation of various PR proteins, which also contribute to systematically acquired resistance $[39,40]$. The PR-1, PR-2 [41], PR-3, PR-4, PR-5 [42], PR-9 [43], PR-10 [44], PR-11 [45], and PR-17 [46] families are associated with acquired resistance to pathogen infections. Among the genes encoding these PR proteins, $P R-1$ is generally considered a marker gene for disease resistance [47]. In this study, PR-related genes were both significantly up- and downregulated in the mutant plants (Table 3), implying that cis-abienol may participate in Tobacco curly shoot virus resistance in tobacco plants. Future research could assess disease resistance in NtCPS2-knockdown and NtCPS2-overexpressing mutants to clarify the contribution of cis-abienol to tobacco disease resistance.

\section{Conclusions}

In this study, a genome-wide transcription profile was obtained for NtCPS2-knockdown tobacco plants edited using CRISPR-Cas9. NtCPS2 is a key gene for cis-abienol biosynthesis in tobacco. Genes involved in the biosynthesis of cis-abienol, early metabolites of GA, and carotenoids (including ABA) were significantly differentially expressed after NtCPS2 knockdown. The expression of PR-related genes also changed in response to low cis- 
abienol contents. Our findings may be useful for further investigation of the molecular mechanisms associated with NtCPS2 gene function and the synthesis of cis-abienol. Additionally, our results can contribute to the development of high-aroma tobacco varieties.

\section{Methods}

\section{Tobacco plant culture and inoculation}

The tobacco plant variety (N. tabacum cv. 8306) used in this study produces high-aroma, flue-cured tobacco with high levels of cis-abienol. Plants were grown in scientific and educational park with loamy tidal soil of Henan Agricultural University, Zhengzhou City, China $(113.63 \mathrm{E}, 37.75 \mathrm{~N})$. Wild-type and transgenic tobacco plants were cultured and grown in mixed soil (1:1 vermiculite:humus) in a growth chamber at $22^{\circ} \mathrm{C}$ with $250-300 \mu \mathrm{mol} / \mathrm{m}^{2} / \mathrm{s}$ photosynthetically available radiation and a 16-h light/8-h dark cycle. Measurements of leaf age started when the length of the middle leaf of each plant reached $1.5 \mathrm{~cm}$. At a leaf age of 60 days, five tobacco plants at the same developmental stage from each group were selected, and the middle leaves were sampled for the measurements of morphological characteristics and RNA extraction. Seeds were collected at 25 days after flowering. Phytophthora nicotianae was cultivated at $24^{\circ} \mathrm{C}$ on clarified V8-Agar, and Zoospores were produced under aseptic conditions [48]. The treatment was applied at the stage around third leaf stage of wildtype and transgenic tobacco plants. Small areas of source leaves were infiltrated with a suspension containing 600-900 zoospores $\mu / \mathrm{L}$ for 7 days [49]. Control tissues were infiltrated with sterile tap water.

\section{Vector construction}

Based on the mRNA sequences and corresponding genome sequences, two CRISPR target sites (Supplementary Table 1) were designed to improve gene-targeting efficiency. Target primers for PCR (Supplementary Table 2) were designed and synthesized. After primer synthesis, fragments containing the target sites were amplified using overlap-extension PCR. The amplified fragments were cloned into a CRISPR expression vector using a recombinant enzyme from Nanjing Novozan Biotechnology Co., Ltd. (Nanjing, China). The CRISPR vector was electroporated into Escherichia coli, and positive clones were screened using colony PCR for Agrobacterium tumefaciens-mediated transformation and tobacco gene transformation.

\section{Agrobacterium-mediated transformation}

Agrobacterium tumefaciens-mediated transformation was performed as follows: $5 \mu \mathrm{L}$ of recombinant plasmid was mixed with $50 \mu \mathrm{L}$ of competent Agrobacterium tumefaciens cells on ice for $30 \mathrm{~min}$. Blank YEB medium was added, and the mixture was incubated at $28^{\circ} \mathrm{C}$ for 12-13 $\mathrm{h}$. Then, the mixture was transferred to YEB solid medium containing $50 \mathrm{mg} / \mathrm{L}$ kanamycin and incubated at $28^{\circ} \mathrm{C}$ for $36-48 \mathrm{~h}$. Mature tobacco seeds were sterilized by washing with $75 \%$ alcohol and $10 \%$ sodium hypochlorite and placed into germination medium. The seeds were then grown under light for 45 days. Samples with a diameter of $0.5 \mathrm{~cm}$ were taken from leaves with a hole punch, transferred to a preculture medium, and incubated for 2 days under light. Agrobacterium tumefaciens was activated in medium containing $50 \mathrm{mg} / \mathrm{L}$ kanamycin. Leaf discs were infected with Agrobacterium tumefaciens, transferred to coculture medium, and left for 3 days. Thereafter, the leaf discs were washed with sterilized distilled water and antibiotics in an aqueous solution. After the leaves were dried, they were transferred to a screening medium and cultured under light. After differentiation, they were transferred to rooting medium. Transformed plants were obtained by rooting culture and transplanted into soil after 1 month.

\section{DNA extraction and sequencing for detecting mutations in the target gene}

Leaflets were collected from each plant, and genomic DNA was extracted using a standard cetrimonium bromide protocol. NPTII-specific primers were used to detect successfully transformed plants via PCR (Supplementary Table 3). After confirming that the exogenous DNA fragment had been inserted, the primer $17 \mathrm{KN} 48$ was designed based on the NtCPS2 gene sequence and target-site location to detect positive plants using PCR. PCR amplification was performed in the following reaction volume: $1 \mu \mathrm{L}$ of DNA, $2 \mu \mathrm{L}$ of $10 \times$ PCR buffer, $0.4 \mu \mathrm{L}$ of dNTP mixture, $0.2 \mu \mathrm{L}$ of forward and reverse primers, $0.2 \mu \mathrm{L}$ of rTaq DNA polymerase (TOYOBO, Osaka, Japan), and $20 \mu \mathrm{L}$ of diethyl pyrocarbonatetreated water. PCR was carried out using the following programme: $94{ }^{\circ} \mathrm{C}$ for $3 \mathrm{~min}, 94^{\circ} \mathrm{C}$ for $30 \mathrm{~s}, 55^{\circ} \mathrm{C}$ for 30 s, $72{ }^{\circ} \mathrm{C}$ for $30 \mathrm{~s}, 72^{\circ} \mathrm{C}$ for $10 \mathrm{~min}$, and $25^{\circ} \mathrm{C}$ for $1 \mathrm{~min}$ for 30 cycles. The PCR products were detected using gel electrophoresis and sequenced.

\section{Measurements of morphological characteristics of transgenic tobacco plants}

Homozygous $\mathrm{T}_{2}$ tobacco plants were selected, and morphological parameters, including plant height, number of leaves, stem girth, internode length, and length and width of the largest leaf, were measured at a leaf age of 60 days. The morphology of leaf glandular trichomes was also characterized. The largest leaves of each plant of the same age were detached, and the epidermis at the centre of each leaf was peeled off to examine the glandular trichomes using an Axioplan 2 microscope (Carl Zeiss, Oberkochen, Germany). The numbers of long and 
short glandular trichomes were counted, and their lengths and diameters were measured. Each seedling had an average of approximately 100 glandular trichomes.

\section{Analysis of diterpenoids in leaf exudates using GC-MS} Leaf exudates were sampled from fresh tobacco leaves, and 1:1 portions of the samples were directly injected into a $6890 \mathrm{~N}$ gas chromatograph coupled to a $5973 \mathrm{~N}$ mass spectrometer (Agilent Technologies, Santa Clara, CA, USA) for GC-MS analysis. Tobacco diterpenoids were identified based on their mass spectra.

\section{RNA-seq analysis}

The middle leaves of wild-type and transgenic tobacco were sampled at a leaf age of 60 days. Total RNA was extracted from frozen leaf samples using TRIzol reagent (Invitrogen, Carlsbad, CA, USA) according to the manufacturer's protocol. RNA integrity was assessed using agarose gel electrophoresis, and RNA purity was checked using a NanoPhotometer ${ }^{\ominus}$ spectrophotometer (Implen, Munich, Germany). RNA concentration was quantified with a Qubit ${ }^{\oplus}$.0 Fluorometer using a Qubit ${ }^{\oplus}$ RNA Assay kit (Life Technologies, Carlsbad, CA, USA). From each qualified sample, $3 \mu \mathrm{g}$ of RNA was sent to Illumina (San Diego, CA, USA) for sequencing. The cDNA library was prepared for sequencing according to the Illumina TruSeqTM RNA Sample Kit protocol. Sequencing was performed using an Illumina HiSeq 2500 system. RNA-seq reads were generated and processed to calculate expression levels, which were averaged over three biological replicates.

\section{Bioinformatics analysis of RNA-seq data}

Raw reads were processed through in-house Perl scripts. Clean reads were obtained by removing adaptercontaining reads, reads containing poly-N, and lowquality reads from the raw reads. The clean reads were then mapped to the tobacco reference genome (ftp:// anonymous@ftp.solgenomics.net/genomes/Nicotiana_ tabacum/assembly/K326). Using Hisat2 v2.0.5 (ftp://ftp. ensembl.org/pub/release-94/gtf/mus_musculus/), an index of the reference genome was built, and paired-end clean reads were aligned to the reference genome. We selected Hisat 2 as the mapping tool because it can generate a database of splice junctions based on the gene model annotation file and thus produce better mapping results than other nonsplice mapping tools. The expression level of each gene was normalized to fragments per kilobase per million for comparison among different samples. Differential expression analysis was performed using the DESeq2 R package (1.16.1) [50], and an absolute $\log _{2}(\mathrm{FC})$ value $>1$ and a corrected $p$-value $<0.05$ were set as the thresholds for DEGs for subsequent analysis.
DEGs were further annotated using GO functional enrichment analysis. GO terms with corrected $p$-values < 0.05 were considered to be significantly enriched for a given DEG. Clusters of orthologous groups and pathway analyses were performed using KEGG (http://www. genome.jp/kegg) analytical tools. We used the clusterProfiler $\mathrm{R}$ package [51] to test the statistical enrichment of KEGG pathways for the DEGs.

\section{Validation of DEGs using qRT-PCR}

The differential expression of 20 genes between wildtype and transgenic tobacco leaf samples was confirmed using qRT-PCR analysis with three biological replicates per sample. Primer sets for the DEGs were designed using Primer Premier 5.0 (Premier Biosoft, San Francisco, CA, USA) and synthesised by Invitrogen Trading (Shanghai) Co., Ltd. (China). All primer sequences are listed in Supplementary Table 4. RNA isolation, cDNA synthesis, qRT-PCR, and statistical analyses were performed as previously described [52]. The expression levels of the DEGs were normalized to that of the internal control gene L25 [53].

\section{Statistical analyses}

Data are presented as the means \pm standard deviations. Two-sample t-tests were used to compare the means between two treatments. Comparisons across multiple treatments were performed using one-way analysis of variance followed by Tukey's honestly significant difference post hoc test with SPSS v19 software (IBM Corporation, Armonk, NY, USA). A value of $p<0.05$ was taken to denote statistical significance.

\section{Abbreviations \\ CPS: Copalyl diphosphate synthase; ABA: Abscisic acid; GC-MS: Gas chromatography-mass spectrometry; ABS: Abienol synthase; RNA-seq: RNA sequencing; qRT-PCR: Quantitative real-time polymerase chain reaction KSL: Kaurene synthase-like; KO: ent-kaurene oxidase; DEGs: Differentially expressed genes; KEGG: Kyoto encyclopedia of genes and genomes; GO: Gene ontology; PSY: Phytoene synthases; LCYs: Lycopene epsilon cyclases; PRs: Pathogenesis-related proteins}

\section{Supplementary Information}

The online version contains supplementary material available at https:/doi. org/10.1186/s12864-021-07796-8.

Additional file 1: Table S1. The sequences of target sites.

Additional file 2: Table S2. The primers for PCR amplification.

Additional file 3: Table S3. NPTII specific primers and 17KN48 target primers.

Additional file 4: Table S4. Primer sequences for qRT-PCR..

Additional file 5: Figure S1. The target sites of NtCPS2.

Additional file 6: Figure S2. Morphological characteristics of mutant and wild-type plants, including plant height (A), internode length (B), number of leaves (C) and girth of stem (D). Values are presented as the means \pm standard deviations ( $n=4$ for leaves and $n=100$ for glandular 
trichomes). Different lowercase letters denote significant differences among plant lines $(p<0.05)$.

Additional file 7: Figure S3. Seedlings of wild-type and transgenic tobacco plants before (A) and after treatment of Phytophthora nicotianae infection for 7 days (B)

\section{Acknowledgments}

We thank Yongle Ding, Zhaoyun Wu, Dongfang Cai, Gang Xue and Qingquan Xu for their help.

\section{Authors' contributions}

Conceptualization, S.X., and T.Y.; methodology, C.C. and L.H.1; software, L.H.2; validation, T.Y.; formal analysis, J.S. and S.X.; investigation, H.L. and T.Y.; data curation, J.S. and S.X.; writing-original draft preparation, S.X.; writing-review and editing, S.X. and T.Y.; funding acquisition, T. Y, S.X., and L.H.1. Revising the manuscript: M.X., L.H.1, L.L., J.Y., W.Z., Z.Z., Q. L and S.X. All authors have read and agreed to the published version of the manuscript.

\section{Funding}

This research was funded by China National Tobacco Corporation Henan company ([2021]27), Henan Tobacco Corporation XuChang Company (2020411000240069), Guizhou Tobacco Corporation Guiyang company (2020-07), Hunan Tobacco Corporation Changsha Company (21-23A04), Guangxi Zhuang Autonomous Region Tobacco Corporation Baise Company(2021-4)

\section{Availability of data and materials}

The online version contains supplementary material available at https://www. ncbi.nlm.nih.gov/bioproject/?term=prjna734477, numbered PRJNA734477 in Nicotiana tabacum Raw sequence reads (TaxID: 4097).

\section{Declarations}

\section{Ethics approval and consent to participate}

Not applicable.

\section{Consent for publication}

Not applicable.

\section{Competing interests}

The authors declare no conflict of interest.

\section{Author details}

${ }^{1}$ College of Tobacco Science, Henan Agricultural University, National Tobacco Cultivation \& Physiology \& Biochemistry Research Centre, Scientific Observation and Experiment Station of Henan, Ministry of Agriculture, Zhengzhou 450002, China. ${ }^{2}$ Technology Center, China Tobacco Zhejiang Industry Co, Ltd., Hangzhou 310008, China. ${ }^{3}$ China National Tobacco Corporation Henan company, Zhengzhou 450002, Henan, China. ${ }^{4}$ Hunan Tobacco Corporation Changsha Company, Changsha 410007, Hunan, China ${ }^{5}$ Guangxi Zhuang Autonomous Region Tobacco Corporation Baise Company, Baise 533000, Guangxi, China.

Received: 26 September 2020 Accepted: 10 June 2021 Published online: 23 June 2021

\section{References}

1. Popova V, Ivanova T, Prokopov T, Nikolova M, Stoyanova A, Zheljazkov VD. Carotenoid-related volatile compounds of tobacco (Nicotiana tabacum L.) essential oils. Molecules. 2019;24(19):3446.

2. Wagner GJ. Elucidation of the functions of genes central to diterpene metabolism in tobacco trichomes using posttranscriptional gene silencing Planta. 2003;216(4):686-91.

3. Yan N, Du Y, Liu X, Zhang HB, Liu YH, Zhang P, et al. Chemical structures, biosynthesis, bioactivities, biocatalysis and semisynthesis of tobacco cembranoids: An overview. Ind Crop Prod. 2016;83:66-80. https://doi.org/1 0.1016/j.indcrop.2015.12.031.

4. Koyama H, Kaku Y, Ohno M. Synthesis of ambrox from L-abietic acid. Tetrahedron Lett. 1987;28(25):2863-6. https://doi.org/10.1016/S0040-403 9(00)96229-4.
5. Barrero AF, Alvarez-Manzaneda EJ, Altarejos J, Salido S, Ramos JM. Synthesis of ambrox(R) from (-)-sclareol and (+)-cis-abienol. Tetrahedron. 1993;49(45): 10405-12. https://doi.org/10.1016/S0040-4020(01)80567-6.

6. Bolster MG, Jansen BJM, Groot $A$. The synthesis of (-)-Ambrox((R)) starting from labdanolic acid. Tetrahedron. 2001;57(26):5657-62. https://doi.org/10.1 016/S0040-4020(01)00493-8.

7. Lin Y, Wagner GJ. Surface disposition and stability of pest-interactive, Trichome-exuded diterpenes and sucrose esters of tobacco. J Chem Ecol. 1994;20(8):1907-21. https://doi.org/10.1007/BF02066232.

8. Kennedy BS, Nielsen MT, Severson RF. Biorationals from nicotiana protect cucumbers against colletotrichum-lagenarium (pass) ell and halst-disease development. J Chem Ecol. 1995;21(2):221-31. https://doi.org/10.1007/BF02 036653.

9. Kennedy BS, Nielsen MT, Severson RF, Sisson VA, Stephenson MK, Jackson DM. Leaf surface chemicals from Nicotiana affecting germination of Peronospora-Tabacina (Adam) sporangia. J Chem Ecol. 1992;18(9):1467-79. https://doi.org/10.1007/BF00993221.

10. Tomita H, Sato M, Kawashima N. Inheritance of labdanoid producing ability in Nicotiana tabacum. Agric Biol Chem. 1980;44:2517-8.

11. Wagner G. Leaf surface chemistry. In: Tobacco Production, Chemistry and Technology. Blackwell Science; 1999. p. 292-303.

12. Kubo T, Sato M, Tomita H, Kawashima N. Identification of the chromosome carrying the gene for cis-abienol production by the use of monosomics in Nicotiana tabacum L. Tob Int. 1982;184(23):57-9.

13. Vontimitta V, Danehower DA, Steede T, Moon HS, Lewis RS. Analysis of a Nicotiana tabacum L.genomic region controlling two leaf surface chemistry traits. J Agric Food Chem. 2010;58(1):294-300. https://doi.org/10.1021/jf9032 $56 h$.

14. Falara V, Pichersky E, Kanellis AK. A Copal-8-ol diphosphate synthase from the angiosperm cistus creticus subsp creticus is a putative key enzyme for the formation of pharmacologically active, oxygen-containing labdane-type diterpenes. Plant Physiol. 2010;154(1):301-10. https://doi.org/10.1104/pp.11 0.159566 .

15. Zerbe $P$, Chiang A, Yuen M, Hamberger B, Hamberger B, Draper JA, et al. Bifunctional cis-abienol synthase from Abies balsamea discovered by transcriptome sequencing and its implications for diterpenoid fragrance production. J Biol Chem. 2012;287(15):12121-31. https://doi.org/10.1074/jbc. M111.317669.

16. Sallaud C, Giacalone C, Töpfer R, Goepfert S, Bakaher N, Rösti S, et al. Characterization of two genes for the biosynthesis of the labdane diterpene Z-abienol in tobacco (Nicotiana tabacum) glandular trichomes. Plant J. 2012; 72(1):1-17. https://doi.org/10.1111/j.1365-313X.2012.05068.x.

17. Carman RM, Duffield AR. The biosynthesis of labdanoids - the optical purity of naturally-occuring manool and abienol. Aust J Chem. 1993;46(7):1105-14. https://doi.org/10.1071/CH9931105.

18. Guo ZH, Wagner GJ. Biosynthesis of labdenediol and sclareol in cell-free extracts from trichomes of Nicotiana glutinosa. Planta. 1995;197(4):627-32.

19. Cui H, Zhang ST, Yang HJ, Ji H, Wang XJ. Gene expression profile analysis of tobacco leaf trichomes. BMC Plant Biol. 2011;11(1):76. https://doi.org/10.11 86/1471-2229-11-76

20. Wang EM, Gan SS, Wagner GJ. Isolation and characterization of the CYP71D16 trichome-specific promoter from Nicotiana tabacum L. J Exp Bot. 2002:53(376):1891-7. https://doi.org/10.1093/jxb/erf054.

21. Severson RF, Arrendale RF, Chortyk OT, Johnson AW, Gwynn GR, Chaplin JF, et al. Quantitation of the major cuticular components from green leaf of different tobacco types. J Agric Food Chem. 1984;32(3):1023-30.

22. Jinek M, Chylinski K, Fonfara I, Hauer M, Doudna JA, Charpentier E. A programmable dual-RNA-guided DNA endonuclease in adaptive bacterial immunity. Science. 2012;337(6096):816-21. https://doi.org/10.1126/ science.1225829.

23. Li JF, Norville JE, Aach J, McCormack M, Zhang D, Bush J, et al. Multiplex and homologous recombination-mediated genome editing in Arabidopsis and Nicotiana benthamiana using guide RNA and Cas9. Nat Biotechnol. 2013:31(8):688-91. https://doi.org/10.1038/nbt.2654.

24. Peng D, Tarleton R. EuPaGDT: a web tool tailored to design CRISPR guide RNAs for eukaryotic pathogens. Microb Genom. 2015;1(4):e000033.

25. Helliwell CA, Sullivan JA, Mould RM, Gray JC, Peacock WJ, Dennis ES. A plastid envelope location of Arabidopsis ent -kaurene oxidase links the plastid and endoplasmic reticulum steps of the gibberellin biosynthesis pathway. Plant J. 2001;28(2):201-8. https://doi.org/10.1046/j.1365-313X.2001. 01150.x 
26. Hedden P, Phillips AL. Gibberellin metabolism: new insights revealed by the genes. Trends Plant Sci. 2000;5(12):523-30. https://doi.org/10.1016/S1360-13 85(00)01790-8.

27. Seo S, Gomi K, Kaku H, Ab H, Seto H, Nakatsu S, et al. Identification of natural diterpenes that inhibit bacterial wilt disease in tobacco, tomato and Arabidopsis. Plant Cell Physiol. 2012;53(8):1432-44. https://doi.org/10.1093/ pcp/pcs085

28. Poovaih BW, Reddy ASN. Calcium and signal transduction in plants. Crit Rev Plant Sci. 1993;12(3):185-211. https://doi.org/10.1080/07352689309701901.

29. Takahashi N, Phinney BO, MacMillan J, editors. Gibberellins. New York: Springer-Verlag; 1991. https://doi.org/10.1007/978-1-4612-3002-1.

30. Yamaguchi S. Gibberellin metabolism and its regulation. Annu Rev Plant Biol. 2008:59(1):225-51. https://doi.org/10.1146/annurev.arplant.59.032607. 092804.

31. Yamaguchi S, Sun TP, Kawaide H, Kamiya Y. The GA2 locus of Arabidopsis thaliana encodes ent-kaurene synthase of gibberellin biosynthesis. Plant Physiol. 1998;116(4):1271-8. https://doi.org/10.1104/pp.116.4.1271.

32. Fleet CM, Yamaguchi S, Hanada A, Kawaide H, David CJ, Kamiya Y, et al. Overexpression of AtCPS and AtKS in Arabidopsis confers increased entkaurene production but no increase in bioactive gibberellins. Plant Physiol. 2003;132(2):830-9. https://doi.org/10.1104/pp.103.021725

33. Hedden P, Phillips AL, Rojas MC, Carrera E, Tudzynski B. Gibberellin biosynthesis in plants and fungi: a case of convergent evolution? J Plant Growth Regul. 2002;20:319-31.

34. Severson R, Johnson A, Jackson D. Cuticular constituents of tobacco: factors affecting their production and their role in insect and disease resistance and smoke quality. Recent Adv Tobacco Sci. 1985;11:105-74.

35. Steede WT, Ma JM, Eickholt DP, Drake-Stowe KE, Kernodle SP, Shew HD, et al. The tobacco trichome exudate $z$-abienol and its relationship with plant resistance to Phytophthora nicotianae. Plant Dis. 2017;101(7):1214-21. https://doi.org/10.1094/PDIS-10-16-1512-RE.

36. Cheng SS, Chung MJ, Lin CY, Wang YN, Chang ST. Phytochemicals from Cunninghamia konishii Hayata act as antifungal agents. J Agric Food Chem. 2012;60(1):124-8. https://doi.org/10.1021/jf2042196.

37. Kenedy BS, Nnelsen MT, Severson RF, et al. Leaf surface chemicals from Nicotiana affecting gennination of Peronospora tabacina (adam) sporangia. J Chem Ecol. 1992;18(9):1467-78. https://doi.org/10.1007/BF00993221.

38. Vontimitta $V$, Lewis RS. Growth chamber evaluation of a tobacco 'Beinhart $1000^{\prime} \times$ 'Hicks' mapping population for quantitative trait loci affecting resistance to multiple races of Phytophthora nicotianae. Crop Breed Genet. 2012;52:91-8.

39. Van LL, Rep M, Pieterse CM. Significance of inducible defense-related proteins in infected plants. Annu Rev Phytopathol. 2006;44:135-62.

40. Li K, Wu G, Li M, Ma M, Du J, Sun M, et al. Transcriptome analysis of Nicotiana benthamiana infected by tobacco curly shoot virus. Virol J. 2018; 15(1):138. https://doi.org/10.1186/s12985-018-1044-1.

41. Bhat S, Folimonova SY, Cole AB, Ballard KD, Lei Z, Watson BS, et al. Influence of host chloroplast proteins on tobacco mosaic virus accumulation and intercellular movement. Plant Physiol. 2013;161(1):134-47. https://doi.org/1 0.1104/pp.112.207860.

42. Mochizuki T, Ogata Y, Hirata Y, Ohki ST. Quantitative transcriptional changes associated with chlorosis severity in mosaic leaves of tobacco plants infected with cucumber mosaic virus. Mol Plant Pathol. 2014;15(3):242-54. https://doi.org/10.1111/mpp.12081.

43. Xu Y, Zhou XP. Role of rice stripe virus NSvc4 in cell-to-cell movement and symptom development in Nicotiana benthamiana. Front Plant Sci. 2012;3: 269.

44. Kong LF, Wu JX, Lu LN, Xu Y, Zhou XP. Interaction between Rice stripe virus disease-specific protein and host PsbP enhances virus symptoms. Mol Plant. 2014;7(4):691-708. https://doi.org/10.1093/mp/sst158.

45. Balasubramaniam M, Kim BS, Hutchens-Williams HM, Loesch-Fries LS. The photosystem II oxygen-evolving complex protein PsbP interacts with the coat protein of alfalfa mosaic virus and inhibits virus replication. Mol PlantMicrobe Interact. 2014;27(10):1107-18. https://doi.org/10.1094/MPMI-02-140035-R.

46. Bhattacharyya D, Chakraborty S. Chloroplast: the Trojan horse in plant-virus interaction. Mol Plant Pathol. 2017;19:504-18.

47. Ryals JA, Neuenschwander UH, Willits MG, Molina A, Steiner HY, Hunt, M.D Systemic acquired resistance. Plant Cell. 1996;8(10):1809-19. https://doi. org/10.2307/3870231.
48. Von Broembsen SL, Deacon JW. Germination and further zoospore release from zoospore cysts of Phytophthora parasitica. Mycol Res. 1996;100(12): 1498-150. https://doi.org/10.1016/S0953-7562(96)80085-2.

49. Varet H, Brilletquéguen L, Coppée JY, Dillies MA. SARTools: a DESeq2- and EdgeR-based R pipeline for comprehensive differential analysis of RNA-Seq data. PLoS One. 2016;11(6):e0157022. https://doi.org/10.1371/journal.pone.01 57022.

50. Colas V, Conrod S, Venard P, Keller H, Ricci P, Panabieres F. Elicitin genes expressed in vitro by certain tobacco isolates of Phytophthora parasitica are down regulated during compatible interactions. Mol Plant-Microbe Interact. 2001;14(3):326-35. https://doi.org/10.1094/MPMI.2001.14.3.326.

51. Yu G, Wang LG, Han Y, He QY. ClusterProfiler: an R package for comparing biological themes among gene clusters. Omi AJ Integr Biol. 2011;16:284-7.

52. Lu J, Du ZX, Kong J, Chen LN, Qiu YH, Li GF, et al. Transcriptome analysis of Nicotiana tabacum infected by cucumber mosaic virus during systemic symptom development. PLoS One. 2012;7(8):e43447. https://doi.org/10.13 71/journal.pone.0043447.

53. Schmidt GW, Delaneym SK. Stable internal reference genes for normalization of real-time RT-PCR in tobacco (Nicotiana tabacum) during development and abiotic stress. Mol Gen Genomics. 2010;283(3):233-41. https://doi.org/10.1007/s00438-010-0511-1.

\section{Publisher's Note}

Springer Nature remains neutral with regard to jurisdictional claims in published maps and institutional affiliations.
Ready to submit your research? Choose BMC and benefit from:

- fast, convenient online submission

- thorough peer review by experienced researchers in your field

- rapid publication on acceptance

- support for research data, including large and complex data types

- gold Open Access which fosters wider collaboration and increased citations

- maximum visibility for your research: over $100 \mathrm{M}$ website views per year

At BMC, research is always in progress.

Learn more biomedcentral.com/submissions 